\title{
EPÍLOGO
}

\section{DEL MÉTODO CIENTÍFICO AL MÉTODO DE VERIFICACIÓN DE UNA NOTICIA}

Jesús Zamora Bonilla Universidad Nacional de Educación a Distancia

\section{Desmarcándonos de la demarcación}

Hacia el año 1800, William Buckley, un soldado británico de casi dos metros de altura que aún no llegaba a la veintena, fue detenido en Londres (donde se recuperaba de heridas recibidas en las guerras contra Napoleón unos meses atrás) por habérsele hallado acarreando un rollo de paño robado. Su excusa de que simplemente estaba haciéndole un favor a una mujer que le había pedido llevar aquella tela, y de que él ignoraba su origen ilícito, no sirvió ante los inflexibles tribunales de Su Majestad, que lo condenaron nada menos que a un destierro de catorce años en Nueva Gales del Sur, en el sudeste de Australia.

Mientras el barco que lo transportaba hacía una escala en la bahía que rodea a la actual Melbourne (una ciudad que aún no había sido fundada en aquel tiempo), William se unió a un pequeño grupo de convictos que decidió escaparse. Uno de ellos fue herido de un disparo por los guardias que los perseguían, pero Buckley y otros consiguieron huir y alejarse, de modo que la nave acabó partiendo sin ellos. Los fugitivos decidieron dispersarse al principio de su huida, para reducir la probabilidad de que los encontraran, pero, abandonados en un territorio desconocido, aunque de clima suave y lleno de vida silvestre, todos acabaron muriendo de hambre, excepto nuestro protagonista, que, exhausto y también al borde de la muerte por inanición, fue encontrado por un grupo de aborígenes de la tribu Wathaurong. Para gran fortuna de William, en vez de matarlo como solían hacer con todos los extraños que encontraban en su territorio, uno de los aborígenes exclamó que aquel gigante no era otro sino el espíritu de su propio hermano, que había muerto hacía poco y que había regresado entre los vivos. Buckley fue cuidado por la tribu como uno de los suyos y vivió con ellos durante más de treinta años aprendiendo su lengua y sus costumbres, hasta que, en 1836, ya en mitad de la cincuentena, decidió regresar a «la civilización» en la recién fundada Melbourne, donde vivió por veinte años más hasta el fin de sus días. 
Las aventuras de William Buckley, llevadas a un libro en los últimos años de su vida a través del un tal John Morgan (pues William fue siempre analfabeto), son una de las principales fuentes para conocer el mundo de los aborígenes australianos en la época de la colonización británica (Henrich, 2020) pero el motivo por el que las he traído a colación no tiene que ver con las peculiaridades de aquellos pueblos, sino con un aspecto mucho más simple de la historia: incluso en un ambiente de agradable clima mediterráneo, con abundante fauna y vegetación, como es el sureste de Australia, un ser humano occidental abandonado a su suerte tenía muy escasas posibilidades de sobrevivir por sí mismo, por la simple razón de que le resultaría extraordinariamente difícil encontrar con qué alimentarse. Solamente la ayuda de un pueblo que disponía del recurso más necesario para los humanos fue la que pudo salvar a nuestro William Buckley.Y ese recurso no es otra cosa, naturalmente, sino el conocimiento: la tribu de los Wathaurong sabía cómo obtener de su entorno lo que necesitaba para sobrevivir, y nuestro héroe tuvo la suerte de que lo encontrasen a tiempo y de caerles simpático.

En realidad, todos los animales necesitan conocimientos. Algunos de estos los poseen de manera innata; otros requieren un cierto aprendizaje. Pero el rango de conocimientos que pueden obtener los individuos de cualquier otra especie es tremendamente limitado en comparación con los que un miembro cualquiera de cualquier sociedad humana asimila a lo largo de su existencia, y por supuesto, los genes son un repositorio demasiado pequeño como para poder contener una enciclopedia tan enorme, así que, en nuestro caso, es casi despreciable la cantidad de conocimientos que recibimos mediante la vía puramente biológica; el resto, es decir, casi todo, tenemos que aprenderlo.Y, por supuesto, para que tú lo aprendas, alguna persona (que puedes ser tú mismo, pero que por lo general será otra) habrá tenido que descubrirlo. Si entendiésemos por "ciencia» algo así como «la generación de conocimientos transmisibles de modo cultural», entonces sería una trivialidad que el género humano ha producido "ciencia» desde su mismísimo origen, pues no de otra manera habría podido sobrevivir. Pero, por supuesto, resulta preferible restringir el sentido del término "ciencia», de tal manera que solo unos cuantos tipos de conocimientos merezcan ser llamados «conocimiento científico", y que solo unos cuantos tipos de actividades merezcan ser llamadas «ciencias». Los Wathaurong poseían muchos conocimientos, pero no tenían «ciencia». Entonces, ¿qué es lo que hace que la ciencia sea ciencia?

El hecho de que podamos razonablemente distinguir el «conocimiento científico» de otros tipos de «conocimiento» no debe hacernos caer en un error que, 
por desgracia, ha sido muy tradicional en el marco de la filosofia de la ciencia y en el resto de las discusiones sobre este asunto: el de empezar intentando establecer algo así como un criterio de demarcación entre «lo científico» y «lo no-científico» (religión, ideología, pseudociencia, metafisica, poesía...). En realidad, la ciencia es solo un tipo de conocimiento, y aquello que hace que sea conocimiento es mucho más importante que lo que hace que ese conocimiento sea «científico». Si pretendemos separar «la ciencia» de todo lo demás, nos dejaremos fuera todo el acervo de conocimientos que ha permitido sobrevivir, y a menudo prosperar, a miles y miles de sociedades a lo largo de la historia. Me parece fundamental que tengamos este punto muy claro si vamos a tratar de entender las relaciones entre el conocimiento científico, la opinión pública, y el papel mediador entre ambos de los medios de comunicación y las redes sociales: la ciencia no es algo completamente aparte y diferente de lo que hay fuera de ella, sino que es una más de las innumerables actividades que los humanos llevamos a cabo cotidianamente para obtener ese recurso sin el que no podemos vivir y que llamamos "conocimiento", conocimiento que solo en algunos casos es "conocimiento científico", pero que no deja por ello de ser conocimiento.

\section{Información, conocimiento y ciencia}

Un término que podíamos haber utilizado en lugar de «conocimiento» es el de «información». Si no lo he hecho, es porque la información puede ser verdadera o falsa, correcta o incorrecta, mientras que «conocimiento (o saber) falso» es un oxímoron. Es una contradicción afirmar algo así como «Juan sabe que Java es más grande que Sumatra, pero en realidad Sumatra es más grande que Java». En cambio, no cometemos ninguna contradicción al afirmar «me pasaron la información de que Java era más grande que Sumatra, pero en realidad Sumatra es más grande que Java». ${ }^{1}$ De hecho, en nuestro mundo hay por doquier información falsa, y cada vez en mayor cantidad y proporción. El conocimiento es, digamos, la «buena» información, y esto en un doble aspecto: primero, debe ser información correcta, en el simple sentido de que las cosas deben ser como

\footnotetext{
1 Lo cierto es que a veces usamos la palabra «información» en el sentido exclusivo de «información veraz»; pero con la expresión «conocimiento» no suele existir dicha ambigüedad: no hay "conocimiento no veraz», salvo usando el término de manera excesivamente forzada; si decimos algo así como «el conocimiento que teníamos ayer ha dejado de ser válido", lo que queremos realmente decir es que el conocimiento que ayer creíamos tener, en realidad no era tal conocimiento.
} 
afirma tal información (al menos, con el margen de aproximación que consideremos relevante); segundo, debemos tener algún tipo de garantía de que la información es efectivamente correcta, o sea, debemos tener la capacidad de identificarla como correcta con suficiente seguridad.

A lo largo de los milenios, los seres humanos hemos ido acumulando y perfeccionando las prácticas que nos permiten obtener (y transmitir a las siguientes generaciones) esa información útil. Todas las actividades cotidianas, por no hablar de los oficios más expertos, dependen de ello de manera esencial. ¿Cuál es la diferencia, entonces, con eso que denominamos «ciencia», y que, por supuesto, consideramos muy diferente al saber que consiste en saber cuál es el camino a casa de nuestros padres, cuál es el momento adecuado para sembrar la avena, o cómo construir un boomerang? La tesis que quiero defender aquí es que no existe una diferencia cualitativa entre las «maneras de obtener información correcta y garantizada» en la vida cotidiana o en las prácticas "precientíficas", por un lado, y las que son propias de «la ciencia», sino que se trata más bien de una diferencia institucional. La "ciencia» es, sobre todo, una institución expresamente dedicada a la búsqueda, acumulación y transmisión de «información de alta calidad». Cuando clasificamos a algunas sociedades del pasado como «pre-científicas», la diferencia más importante en que debemos fijarnos no debe ser algo así como que la vida en aquellas sociedades se basaba en «creencias precientíficas» (mitos, religiones, leyendas, refranes, etc.), mientras que la nuestra se basaría en el "conocimiento científico», pues, como hemos indicado, la mayor parte de la información que se utilizaba de hecho en esas sociedades tenía que ser inevitablemente "correcta», y por otro lado, tampoco es que las creencias «anticientíficas» hayan dejado de tener presencia e influencia en las sociedades actuales. No, la diferencia importante consiste en que nuestra sociedad posee, al contrario que otras, una institución (o mejor dicho, un sistema de instituciones) específicamente dedicada a la obtención de información de alta calidad, y a la transmisión de dicha información al resto de los ámbitos sociales. En las sociedades anteriores, cada actividad, cada profesión, eran las responsables de ir obteniendo la información necesaria para ellas, y además esa búsqueda de conocimiento no estaba organizada como un fin en sí mismo, sino que era más bien un resultado secundario de las propias prácticas, acumulándose a lo largo de los siglos por ensayo y error o poco más, y la transmisión de ese conocimiento se solía realizar a su vez dentro de dichos ámbitos, sin compartirlo sistemáticamente con otros. Tampoco existía la noción de un tipo de conocimientos que fuesen intrínsecamente «transversales», en el sentido de que no 
pertenecieran a una actividad práctica o un oficio concretos, sino que pudieran ser aplicados a muchos ámbitos a la vez (en este sentido, lo que llamamos conocimiento «abstracto» o «teórico»—palabra griega esta última que significa "contemplativo» - no debe, pues, entenderse tanto como un conocimiento «completamente ajeno a su posible aplicación práctica», sino más bien como un conocimiento cuya aplicación práctica es abierta e indefinida). La «ciencia», pues, surge históricamente cuando algunas personas consideraron que obtener conocimiento "por sí mismo» es una actividad que puede separarse socialmente de las demás; a quienes lo hicieron por primera vez se les llamó «filósofos» (literalmente, «amigos de la sabiduría»), en tiempos de la Grecia clásica, aunque, por supuesto, aquello fue solo la semilla de un crecimiento institucional que durante muchos siglos fue bastante lento y muy limitado espacial y temporalmente, y que solo en los últimos siglos se ha transformado en la compleja realidad que conocemos hoy en día.

\section{3. ¿Existe el «método científico»?}

Naturalmente, no es solo característico de «la ciencia» el hecho de que se haya separado institucionalmente de otras actividades para dedicarse ex profeso a la búsqueda de conocimiento (mientras que en otras dicha búsqueda suele ser un resultado lateral, por así decir), sino el hecho de que, al cobrar de este modo existencia autónoma, ha llegado a ser mucho más eficiente en la producción de información de calidad que el resto de las actividades y prácticas sociales, eficiencia que hemos de entenderla tanto en términos de la cantidad de información producida, como en términos del nivel de garantía y corrección con que la obtiene. Algo, por otro lado, que es un resultado habitual del proceso que conocemos como "división social del trabajo». Los conocimientos científicos suelen ser «mejores» que los no-científicos, no por la aplicación de algo especial que podamos llamar «el método científico», sino sencillamente porque se dedican a ello muchos más recursos, mucha mayor especialización y mucho más cuidado. Es decir, el conocimiento científico suele ser «mejor» conocimiento que el obtenido por otras vías por una razón tan simple como la razón por la que unos zapatos fabricados por un zapatero profesional serán mucho mejores que unos que pudiera fabricar yo con mis torpes manos: por la pericia acumulada por los profesionales correspondientes, más que por la pre-existencia de una especie de algoritmo llamado «el método científico» o 
«el método zapateril» en el mundo de las ideas. Igual que no existe un método de hacer zapatos, sino una enorme variedad, tampoco existe un método para obtener conocimientos científicos, sino que lo que tiene que hacer, pongamos, un arqueólogo para averiguar cómo era la sociedad correspondiente a cierto yacimiento es radicalmente distinto a lo que tiene que hacer un químico para descubrir la estructura de una cierta molécula, o a lo que tiene que hacer un epidemiólogo para determinar la mejor forma de evitar la propagación de una pandemia.Y ni siquiera en el caso de una de estas especialidades considerada aisladamente es razonable encontrar una lista cerrada de «métodos», sino que estos siempre están evolucionando y siendo sometidos a discusión, igual que las formas de fabricar zapatos.

Ahora bien, igual que todos los zapatos, y todas las maneras de producirlos, están condicionadas en último término por la forma y la función de nuestros pies, también todas las formas de obtener conocimiento (tanto el «científico», como el "extra-científico») están en el fondo determinadas por la propia naturaleza de la información y de nuestras capacidades de procesarla, y quizá una mínima descripción de estos condicionantes puede ser presentada como una especie de "resumen del método científico» (un resumen más bien caricaturesco, pero que, como toda buena caricatura, puede capturar ciertos elementos esenciales de aquel sujeto al que representa). Los dos elementos principales de esa caricatura serían:

1. Casi todo nuestro conocimiento depende en último término de lo que observamos a través de nuestros sentidos; o, por decirlo de otro modo, la experiencia es el principal «lugar de entrada» de la información en nuestro acervo de conocimientos.

2. Las proposiciones que describen o intentan describir los hechos están relacionadas entre sí por vínculos inferenciales, en el sentido de que algunas proposiciones se siguen de otras (si A es verdad, entonces B tiene que ser verdad) o son incompatibles con otras (si A es verdad, entonces $\mathrm{C}$ no puede ser verdad), aunque esta relación es a menudo meramente probabilística (si A es verdad, entonces es probable que $\mathrm{B}$ sea verdad, o que C no lo sea). Dicho de otra manera: los conocimientos deben organizarse y justificarse mediante el razonamiento lógico.

Otro aspecto esencial de toda actividad productora de conocimiento, socialmente relevante, es el hecho de que ni la «observación empírica» ni el 
«razonamiento lógico» que se mencionan en los dos puntos anteriores suelen poder llevarse a cabo en solitario: por lo general, no tenemos más remedio que basarnos en lo que han observado otras personas, no nosotros mismos con nuestros propios ojos; y por lo general, los argumentos a favor o en contra de una determinada tesis tienen más la forma de una discusión entre varias personas que la de un razonamiento elaborado de principio a fin por una sola persona (de hecho, parece que nuestras capacidades de razonamiento están mucho mejor adaptadas biológicamente a la situación que podemos llamar «defender nuestra propia opinión en un debate público», que a la situación que llamaríamos «reflexionar objetivamente en la soledad de nuestro gabinete») (Mercier y Sperber, 2017).

El papel fundamental que la observación y la inferencia tienen en la generación de información de alta calidad ha llevado a dos concepciones tradicionales sobre el «método científico» que se caracterizan por priorizar de manera absoluta una sola de estas dos fuentes. Por un lado, tendríamos el inductivismo, según el cual el método científico sería el «método inductivo»: hacer observaciones lo más completas y sistemáticas posibles, para inferir de ellas, por simple generalización (o «inducción»), las leyes o regularidades ejemplificadas en lo que hemos observado. Supuestos defensores de algo parecido a este método habrían sido Aristóteles, Francis Bacon o Stuart Mill, y quizá los «positivistas lógicos» de la primera mitad del siglo Xx. Por el otro lado tendríamos el deductivismo, según el cual la ciencia debe seguir el «método deductivo», partiendo de principios racionales intuitivamente verdaderos, e infiriendo a partir de ellos, por argumentos puramente lógicos o matemáticos, las leyes que deben gobernar de modo inevitable la naturaleza; solo al final del proceso habría un último escalón que permitiría deducir de tales leyes lo que necesariamente se observará cuando se mire el mundo con cuidado a través de mediciones o experimentos. Los héroes imaginarios de esta visión de la ciencia habrían sido Platón, Descartes, Leibniz o Hegel, y algo similar sería lo que habría defendido en nuestra época algún que otro fisico matemático, como David Deutsch (2011).

Inductivismo y deductivismo (o sus versiones más filosóficas, «empirismo» y «racionalismo») serían, como digo, dos caricaturas que incluso los autores que he mencionado no defienden, por supuesto, de un modo tan simplista y exagerado como el que he retratado. En realidad, en todo proceso de investigación científica (salvo, quizá, en las matemáticas puras, y no siempre) hay abundantes «momentos inductivos» (de recopilación de datos y obtención de 
regularidades a partir de ellos) y «momentos deductivos» (de argumentación puramente lógica basada en principios abstractos, sin prestar aparentemente gran atención a los datos empíricos), y cada disciplina va desarrollando técnicas, normas o hábitos (incluso incompatibles entre sí) sobre cuándo utilizar más los unos o los otros y cómo llevarlos a cabo. Pero hay otro elemento fundamental en la investigación científica (y en muchas otras formas de obtención de conocimiento, pero en esta singularmente) que no hemos indicado todavía, y que tiene que ver con el hecho de que muchas de las entidades, propiedades, sistemas, etc., a las que se refieren las proposiciones científicas son cosas que no resultan en absoluto aparentes en los datos observables, ni tampoco asoman de manera clara en los «principios racionales» de los que se jacta el deductivismo. Los conceptos y relaciones que describen a tales entidades «escondidas» no hay más remedio que inventárselos, concebirlos en nuestra mente como una simple conjetura, con la esperanza de que esa conjetura podamos enlazarla de manera más o menos fructífera con las redes de argumentación que las conectarán "hacia abajo» (con los datos empíricos) y «hacia arriba» (con los principios racionales).

Estas conjeturas suelen recibir el nombre de hipótesis, o a veces, teorías o modelos, y, aunque la variedad de los métodos relacionados con ellas es incluso mayor que la que hay con los dos elementos considerados más arriba (observación y razonamiento lógico), lo cierto es que también en este caso su estructura determina al menos algunos aspectos básicos del modo como pueden ser sometidos a crítica para que vayan avanzando en el camino que las lleva, de simples conjeturas, a verdaderos conocimientos. Me refiero a lo que suele conocerse como método hipotético-deductivo: podemos esforzarnos en deducir, mediante argumentos lógicos, qué hechos en-principio-observables tendrían que ser verdaderos en el caso de que la hipótesis fuese cierta (este es el elemento "deductivo", al que, si tales hechos aún no sabemos si son ciertos o no, llamamos predicción), para, posteriormente esforzarnos en determinar empíricamente si tales hechos en-principio-observables se observan o no, en las condiciones o circunstancias determinadas por la hipótesis. Si no se observan, si observamos que el hecho predicho no ocurre, entonces la lógica nos proporciona un argumento en contra de nuestra hipótesis, y habremos de rechazarla, o al menos modificarla. Si se observan, eso no demuestra de modo concluyente que la hipótesis sea verdadera (pues futuras predicciones realizadas a partir de ella, e independientes de las que hemos observado ahora, podrían fracasar), pero al menos suponen una razón a favor de la conjetura. 
El problema con las hipótesis (además de que no podemos observar directamente si se cumplen o no, ni podemos deducirlas como teoremas necesariamente válidos a partir de los "principios racionales») es que, como surgen de nuestra imaginación, podemos inventarnos infinidad de ellas (lo que, por sí mismo, no es malo), y a menudo sucede que tenemos varias conjeturas que son igual de coherentes con los hechos observados (esto es lo que se llama «el problema de la infradeterminación empírica de las teorías»). En ese caso, tenemos que utilizar algunos otros tipos adicionales de razonamiento para decidir cuál de todas esas conjeturas es preferible; el más importante de los cuales es el que se conoce como principio de parsimonia o «navaja de Ockham»: a igual apoyo empírico, la hipótesis más simple es la que tiene mayor probabilidad de ser correcta. El problema, naturalmente, es que dicha «simplicidad» puede ser valorada de maneras muy distintas según las circunstancias, aunque menudo podemos identificar dicha simplicidad con la coherencia con el resto de nuestro conocimiento: la hipótesis que nos obligue a hacer menos conjeturas adicionales, o menos revisiones sobre lo que pensábamos conocer, será la preferible.

\section{Otros buscadores de conocimiento}

En resumen, los científicos siguen métodos que no se diferencian mucho (salvo porque están mucho más refinados, elaborados, y adaptados a los ámbitos y tecnologías relevantes en cada caso) de los que se utilizan en cualquier otro ámbito de la vida para adquirir conocimientos con los que resolver nuestros problemas: observar con cuidado, razonar con cuidado, formular hipótesis, contrastarlas, y quedarnos con las explicaciones más simples posibles de los hechos que hemos llegado a descubrir. La diferencia principal entre los científicos y otras personas que también necesitan «averiguar cosas» es, como decíamos más arriba, que los primeros hacen de esto su actividad principal, mientras que en casi todos los demás casos la búsqueda de conocimientos es un paso entre muchos otros (y a veces bastante secundario) en el camino a finalidades más prácticas. Pero lo cierto es que hay algunas profesiones en las que la búsqueda de conocimiento sí que es una tarea tan básica y fundamental como en la ciencia: pensemos, por ejemplo, en los tribunales (y las fuerzas de policía que los ayudan a investigar los delitos), o en los espías, exploradores y otros «servicios de inteligencia». El «método» en todos estos casos se reduce a lo mismo: observar, conjeturar y razonar con el mayor cuidado posible, aunque a menudo las «ob- 
servaciones» consisten en obtener el testimonio de otras personas que son las que en efecto han observado los hechos relevantes.Y, por supuesto, detectives y rastreadores no suelen tener, o no con la misma frecuencia, el problema que a menudo tienen los científicos de intentar averiguar cómo funcionan cosas que, propiamente hablando, son inobservables, como las órbitas de los planetas, los campos electromagnéticos, o las moléculas.

Espías, exploradores, inquisidores y jueces los ha habido desde hace milenios, pero en los últimos dos siglos ha surgido también otra profesión en la que la búsqueda de conocimientos es un elemento esencial: el periodismo. Naturalmente, una diferencia importante entre los espías, detectives y periodistas, por un lado, y los científicos, por otro, es que los segundos intentan por lo general obtener conocimientos que aún no existen, mientras que los primeros suelen intentar averiguar cosas que sí que hay alguien que sabe ya. Pero lo cierto es que a la información le da lo mismo (por lo general) cuánta gente la posea, y si tú no la tienes, el tipo de cosas que tienes que hacer para adquirirla, si quienes sí que la poseen no te la quieren contar, es bastante parecido a las que debería seguir un científico: comparemos, por ejemplo, el caso de un periodista investigando un episodio de corrupción del partido político gobernante, con el caso de un historiador haciendo lo mismo pero con quienes gobernaban hace siglo y medio. En ambos casos se trata de acumular pruebas, tantear hipótesis, y razonar sobre la coherencia o incoherencia entre cada pieza de información, hasta dar con una imagen global en la que todo encaje lo mejor posible. Por lo tanto, el científico y el periodista, al menos el "periodista de investigación», no se diferencian mucho cualitativamente hablando, en cuanto a su propia actividad. Ambos deben formular la mejor teoría posible sobre el problema acerca del cual estén investigando, deben acumular hechos desconocidos que corroboren o debiliten cada hipótesis alternativa, deben prestar atención a la plausibilidad de cada idea según su coherencia con el resto de los hechos conocidos, y deben presentarla con la mayor claridad y rigor posibles.

En cambio, las diferencias entre el científico y el periodista son más que notables cuando tenemos en cuenta no solo su propia actividad «individual», sino su relación con otros agentes. La diferencia más evidente se refiere a la audiencia de cada uno: el científico suele escribir sus trabajos pensando en otros colegas, que son quienes van a evaluarlo y quienes van a decidir, en último término, si las conclusiones a las que ha llegado hay que considerarlas como suficientemente correctas o no. El periodista, en cambio, escribe para «el público», y es el juicio 
del público el que desempeña el papel más parecido al de «evaluación» (en este caso, no "por pares», como en la ciencia) de lo que el periodista publica. Los científicos también escriben a veces "para el público", p. ej. cuando crean obras de divulgación, pero en ese caso su tarea no consiste en presentar unos determinados conocimientos para que sean evaluados por el público, pues la verdadera «evaluación» de este conocimiento ha sido realizada previamente por la comunidad científica, y lo mismo ocurre, por supuesto, cuando son los periodistas los que realizan trabajos de divulgación (no de investigación); en cambio, el trabajo de investigación del periodista sí que es sometido al juicio del público, que lo tomará (o lo rechazará, o unos una cosa y otros la otra) como un elemento que forme parte de su opinión sobre el ámbito al que pertenece la realidad investigada.

La segunda diferencia importante en cuanto a la relación de científicos y periodistas con personas ajenas a su profesión tiene que ver con el hecho de que, por lo general, lo que dicen los científicos no suele tener graves repercusiones prácticas directas para quienes no se dedican a la ciencia (aunque, por supuesto, hay excepciones, y, también por supuesto, las repercusiones indirectas son con frecuencia muy significativas); en cambio, los asuntos de los que hablan o escriben los periodistas suelen ser en la inmensa mayoría de los casos acciones de individuos, empresas o instituciones concretas, con nombre y apellidos, y estos sujetos tienen un interés inmediato en que los mensajes periodísticos sean favorables para ellos, o al menos no sean perjudiciales. Hay un dicho muy conocido según el cual la diferencia entre la divulgación y el periodismo es que la divulgación consiste en explicar de manera sencilla cosas difíciles de comprender que nadie pretende que se mantengan ignoradas, mientras que el periodismo consiste en contar cosas sencillísimas de entender, pero que alguien no quiere que se sepan. En este sentido, normalmente los artículos científicos están mucho más cerca de lo primero que de lo segundo (aunque prescinden de lo de «explicar de manera sencilla»). Esta diferencia es tan importante que nos lleva de cabeza al siguiente apartado, con el que cerraremos este capítulo.

\section{Ciencia, periodismo, intereses e ideologías}

Exactamente igual que la función primordial de la agricultura es la de producir alimentos de la manera más eficaz posible, la función primordial de la ciencia y del periodismo no es otra que la de producir y distribuir información del modo más eficaz posible. Naturalmente, los diversos valores, intereses y 
cuotas de poder de los miembros de la sociedad influirán en qué alimentos son producidos en mayor o menor cantidad, y quiénes se beneficiarán más o menos de ellos, y de modo similar, esos mismos valores, intereses y cuotas de poder influirán en qué tipos de investigaciones recibirán más recursos y quiénes serán los principales beneficiarios de sus resultados y de su divulgación y aplicación. Es razonable esperar que alguien prefiera que el conocimiento avance más en un determinado terreno que en otros (compartamos tales preferencias o no los demás), pero lo que no es razonable es esperar que alguien prefiera que, sobre ese asunto en el que tiene gran interés, la información que se obtenga sea de mala calidad, antes que información correcta.

Los seres humanos, por desgracia, estamos sometidos a numerosos sesgos que a menudo nos hacen percibir y entender las cosas de manera bastante alejada de la verdad (ver, por ejemplo: Matute, 2019), pero, como hemos visto más arriba, la característica principal de la ciencia consiste en el esfuerzo por obtener información con la mayor garantía posible de corrección, es decir, en el esfuerzo por corregir todas aquellas tendencias que nos llevan a cometer errores al intentar averiguar cómo son las cosas en un determinado ámbito. Hay, por supuesto, numerosas razones por las que tal esfuerzo puede a veces no dar como resultado una información de calidad realmente elevada sobre la que se haya podido construir un consenso científico bien fundamentado, en especial la propia complejidad intrínseca de algunos ámbitos del universo en comparación con otros, o la dificultad para encontrar datos abundantes y fiables relativos a ellos. Esto conduce de modo inevitable a que ciertas áreas de la ciencia parezcan más «científicas» $\mathrm{u}$ «objetivas» que otras, aunque, en realidad, en todas las disciplinas científicas suele ocurrir que los temas de investigación que se hallan más «en la frontera del conocimiento» son asuntos en los que predomina el debate por encima del consenso, precisamente porque todavía no se sabe lo suficiente sobre ellos como para haber podido descartar todas las posibles teorías excepto una.

El periodismo, por el contrario, carece de algo parecido al «mecanismo de autocorrección» que existe en la ciencia, y que básicamente consiste en el compromiso de los científicos por someter a prueba lo más duramente posible cada teoría que se proponga, y en aceptar la que mejor resista aquellas pruebas, aunque no sea la que a un científico en concreto «le interesaba» que se aceptase. Esto se debe a que, como veíamos más arriba, los evaluadores últimos de la tarea del periodista no son sus colegas, sino el público, y el público carece de un interés tan intenso como el de los científicos por la calidad objetiva de 
la información que termina aceptando. Ahora bien, ¿cómo es esto posible? ¿Cómo puedes tú, como lector o espectador, no estar «suficientemente interesado» en que tus opiniones sobre los temas acerca de los cuales te informas sean opiniones correctas mejor que opiniones incorrectas? Volveremos a esta cuestión enseguida, pero antes permítaseme introducir el otro gran factor que hace que la información periodística no tenga por lo general el mismo grado de objetividad que el conocimiento científico, y que tiene que ver con lo que veíamos al final del apartado anterior: como acabamos de decir, es razonable esperar que las personas prefieran tener información correcta antes que información incorrecta, pero eso es muy distinto a la cuestión de si prefieren que los demás tengan buena o mala información. Si los beneficios de una empresa, o los votos que reciba un partido, dependen en gran medida de que los consumidores o los votantes estén equivocados sobre ciertos asuntos, lo lógico es que aquella empresa o aquel partido político tengan un interés en generar desinformación sobre esos temas, es decir, «información incorrecta», o al menos, información que lleva al público a tomar decisiones que no coinciden con las que habría tomado en caso de contar con información objetivamente mejor. La mejor defensa contra esto es fomentar la libre competencia entre empresas, entre partidos, y entre medios de comunicación, para que el público pueda comparar aquella información incorrecta con otras fuentes, además de establecer cuantos controles de ética y pluralidad informativas sea posible a todos los niveles, aunque también existe el riesgo de que algunos de estos «controles» degeneren en una especie de censura, lo que demuestra que el equilibrio óptimo en esta materia es dificil de obtener, e inclusive de definir. Por otro lado, tampoco hay que engañarse pensando que la propia actividad científica está completamente libre de la influencia de agentes a los que les resulte beneficiosa la producción y difusión de «desinformación», pero en este caso los mecanismos de control interno de la ciencia, con las graves repercusiones que para un científico individual puede tener el verse involucrado en un caso de fraude, parecen al menos más eficientes que en los medios que se dirigen a la opinión pública.

También hay que mencionar los casos en los que son los propios intereses académicos de los científicos los que pueden llevarles a no perseguir la verdad con el suficiente rigor: al fin y al cabo, la institución científica funciona otorgando grandes recompensas a quienes son reconocidos como los descubridores de la mejor solución a un problema científico, y esto puede llevar a que un investigador prefiera presentar sus datos y argumentos de tal modo que parezcan más favorables para su propia teoría que lo que objetivamente puede justificarse. 
Esto puede ocurrir incluso al nivel colectivo de toda una disciplina científica o una "escuela» dentro de ella: un grupo de investigadores pueden acabar siendo "ciegos» a algunos argumentos decisivos en contra de la teoría (o "paradigma», por utilizar un viejo término) (Kuhn, 1962) de la que por motivos históricos dependen sus carreras. Esto conduce a un dilema parecido al que hemos señalado de pasada respecto a los medios de comunicación, sobre cuánto fomentar la diversidad frente a cuánto valorar el consenso, y no voy a engañar a nadie aquí afirmando que los filósofos de la ciencia hayamos descubierto una respuesta satisfactoria a tal dilema (ver: Kitcher, 2003).

Un factor que puede tener causas y efectos similares, pero que no es idéntico, al de la influencia de los intereses en la objetividad y calidad de la información que se difunde en los medios de comunicación (y en parte, aunque seguramente mucho menos, en la investigación científica) es el que conocemos como ideología. En cierto sentido, la ideología podemos entenderla como un tipo de sesgo más, o como un conjunto de sesgos, y también como algo influido por los propios intereses (o por los intereses «de clase», según la vieja tradición marxista), pues uno de los elementos característicos de las ideologías es el hecho de que funcionan principalmente como mecanismo de auto-justificación de las decisiones o costumbres de un determinado grupo (o, podríamos decir, como «calmantes de la disonancia cognitiva»). Pero lo más característico de la ideología es, seguramente, su íntima conexión con la política, de tal manera que «ideología» e «ideología política» nos resultan prácticamente sinónimos, y en el mundo de los medios de comunicación, la ideología desempeña un papel bastante más central que otros tipos de sesgos, hasta el punto de, en la mayoría de las ocasiones, definir la «identidad política» de cada medio. Esto lleva a una desagradable característica de estos medios: el hecho de que, en gran medida, más que funcionar como fuentes de información objetiva (como proveedores de respuestas razonablemente correctas a las preguntas del tipo «¿qué es lo más importante que ha pasado en las últimas horas?»), parecen hacerlo como suministradores de auto-complacencia ideológica (proveedores de respuestas a preguntas del tipo «¿cómo se puede interpretar lo que ha pasado en las últimas horas de tal manera que me confirme lo mejor posible mis simpatías y antipatías políticas?»). El hecho de que cada uno de nosotros prefiramos informarnos en unos medios de comunicación mejor que en otros, no tanto porque la información que ofrecen aquellos sean objetivamente mejor que la que ofrecen estos, sino porque tengamos más facilidad para aceptarla como verídica por estar más de acuerdo con nuestra ideología política (porque en ella encontramos más - y más ponzoñosas - críticas a los políticos que consideramos como 
"adversarios»), es algo seguramente tan inevitable como malsano. No tengo claro que las redes sociales hayan exacerbado esta situación con respecto a como podría ser a lo largo del siglo Xx, cuando la comunicación entre los medios y el público era básicamente unidireccional; pero sin duda esas redes constituyen también un caldo de cultivo propicio para la circulación de información que es aceptada por ser coherente con nuestra ideología, más que por su objetividad.

Esto último me lleva a la reflexión con la que terminará el capítulo: en los últimos tiempos, y quizás en este caso sí que mucho más por el efecto de la «democratización» de la comunicación gracias a las redes sociales y a otras plataformas informáticas, se ha incrementado la fuerza de otro factor que tiende a erosionar la calidad de la información que circula entre el público, y que no es otro que lo que el filósofo Harry Frankfurt denominó «pamplinas» (bullshit) (Frankfurt, 2006). Estas no son tanto el intento deliberado de difundir información falsa, para engañar al público o para reforzar una posición política, sino más bien el mero desprecio a la verdad, el intento de adquirir notoriedad mediante la difusión de información estúpida, simplemente porque la notoriedad así adquirida es mucho más valiosa para quien la persigue que la calidad de la información, y porque lo chocante y disruptivo de esa información le hace sentirse, al público que la recibe y acepta, como alguien que está «por encima de la masa crédula».

Naturalmente, todos estos factores que juegan en contra de la objetividad de la información tal como se ofrece en los medios de comunicación no son excluyentes entre sí, sino que suelen ir acompañados unos de otros en diversas proporciones, y, como decía un poco más arriba, seguramente es imposible eliminarlos en completo, y ni siquiera en una medida apreciable. Por tanto, si este breve texto mío contribuye aunque sea en un pequeñísimo porcentaje a que sus lectores sean menos proclives a sentirse atraídos por tales cantos de sirena de los intereses, los sesgos, la ideología y el bullshit, tanto si lo hacen como periodistas, como si lo hacen en cuanto usuarios de los medios de comunicación, habrá valido la pena el escribirlo.

\section{Bibliografía}

Frankfurt, H. (2006) On bullshit. Princeton, NJ: Princeton University Press. HeNRICH, J. (2020). The WEIRDest people in the world: how the West became psychologically peculiar and particularly prosperous. NY, NY: Macmillan Publishers. 
Matute, H. (2019), Nuestra mente nos engaña. Barcelona, ES: Shackleton Books.

Kitcher, P. (2003). Science, truth, and democracy. Oxford, UK: Oxford University Press.

Kunn, T. S. (1962), The Structure of Scientific Revolutions. Chicago, IL:The University of Chicago Press.

Mercier, H., \& Sperber, D. (2017). The enigma of reason. Cambridge, MA: Harvard University Press.

Jesús Zamora Bonilla (Madrid, 1963) es actualmente decano de la facultad de Filosofia de la UNED, y catedrático de filosofia de la ciencia en dicha facultad, así como doctor en Filosofia y en Ciencias Económicas. Es autor de más de una docena de libros, incluyendo tanto obras filosóficas como literarias, y asimismo ha publicado más de doscientos artículos de investigación, de divulgación y de opinión. Es también un activo divulgador en internet y redes sociales, destacando sus blogs $A$ bordo del Otto Neurath y Escritos sobre gustos, y sus colaboraciones periódicas en el blog de divulgación de la Cátedra de Cultura Científica de la Universidad del País Vasco, Mapping Ignorance. En el campo de la comunicación científica, destaca su actividad como impulsor y director del Máster en Periodismo y Comunicación Científica de la UNED, así como la creación de la web DivulgaUNED.

Página web: https://www2.uned.es/dpto_log/jpzb/ 

